\title{
Power flows in multi-junction power supply circuits
}

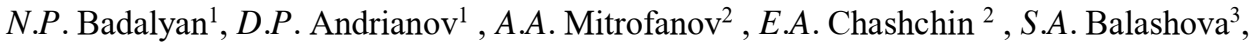 \\ and G.V. Maslakova ${ }^{4, *}$ \\ ${ }^{1}$ Vladimir State University named after Alexander and Nikolay Stoletovs, 600000 Vladimir, Russian \\ Federation \\ ${ }^{2}$ Kovrov State Technological Academy named after V.A. Degtyarev, 601910 Kovrov, Russian \\ Federation \\ ${ }^{3}$ Joint-stock company "All-Russian research Institute "Signal», 601903 Kovrov, Russian Federation \\ ${ }^{4}$ Lipetsk State Technical University, 398055 Lipetsk, Russian Federation
}

\begin{abstract}
The article discusses a methodology that allows for an accurate calculation of the parameters of electrical equipment from the point of view of optimizing the power supply system. Taking into account the characteristics of transients processes makes it possible to select equipment in maximum load modes. Modeling of power flows caused by changes in the load of consumers is carried out on the example of calculating the power supply circuit of five junctions. It is shown that it is possible to change the direction of flows of transmitted electric power at certain ratios of parameters in individual elements of the circuit. Purpose of research: to show the possibility of changing the picture of the transmitted capacities by the elements of the power supply system, depending on consumer loads.
\end{abstract}

\section{Introduction}

Analysis of the components of the energy market [1] shows the need for greater flexibility of power supply systems.

On the one hand, the importance of solving the problem of storing electricity in storage devices is increasing [2]. The study of the implementation of energy storage systems in municipal economy [3] has shown the potential of this approach. On the other hand, the issues of optimal use of power supply systems [4], which cover the generation, transmission, and consumption of electric power, are getting serious importance. Multipurpose optimization of power losses of complex energy systems requires the development of evolutionary calculation methods [5] and adaptive control algorithms [6].

Dispatching of the flows of transmitted electric power has become one of the key problems of energy supply at present [7].

In this regard, the issues of modeling power supply systems are of particular importance.

There are three global levels of problems that designers have to solve:

\footnotetext{
*Corresponding author: maslakova.gv@ya.ru
} 
- description of objects: the number and location of energy sources and consumers, their characteristics, topology and connection parameters;

- mathematical calculation methods and resources of computing systems;

- operating conditions of power systems: nature and intensity of the load.

The transfer of power supply to digital management (digitalization) as the nearest prospect for the development of the technical level is impossible without an objective assessment of the state of power systems, which is based on a clear definition of operating parameters.

The mathematical apparatus that provides reliability of calculations and substantiation of system parameters becomes essential. An overview of mathematical methods that have found application in modeling processes in electrical systems is presented in $[8,9]$.

Modern technical literature shows great attention to modeling and optimization of parameters of multi-junction power supply circuits [10-16], which have from 5 to 57 or more junctions.

It should also be noted that the parameters of real elements of power supply systems are stochastic, which imposes certain difficulties on the representation of electrical loads [1719].

\section{Preliminary remark}

Considering instantaneous values of current, voltage, and power allows us to take into account the maximum possible jumps of these parameters. Taking into account extreme values of parameters makes it possible to calculate the equipment in conditions of possible overload, evaluate its longevity and performance.

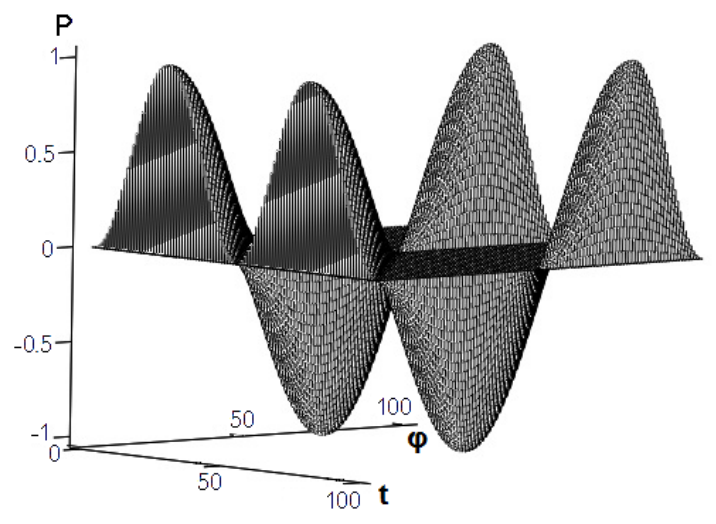

Fig. 1. The graph of change of instantaneous power.

It should be noted that the consideration of instantaneous power characteristics causes certain difficulties. A graph that shows the generalized dependence of the power value change on the phase shift between sinusoidal voltage and current strength can be an example of this (Fig. 1). The specified graph depends on two parameters and takes into account the dependence of power $\mathrm{P}$ on time $\mathrm{t}$ and the phase shift between voltage and current $\varphi$. The process is considered for a period of time equal to one period or $2 \pi$ radians. The phase shift $\varphi$ also changes within a single period or $2 \pi$ radians. 


\section{The method}

The object of the study was a power supply circuit with two sources of electricity (electromotive force), 5 consumers with active-inductive and capacitive loads, and 7 chains (active-inductive load corresponding to the parameters of power lines) (Fig. 2). Power sources synchronously generate sinusoidal alternating electric current of standard industrial frequency. The scheme has a ring structure of connections.

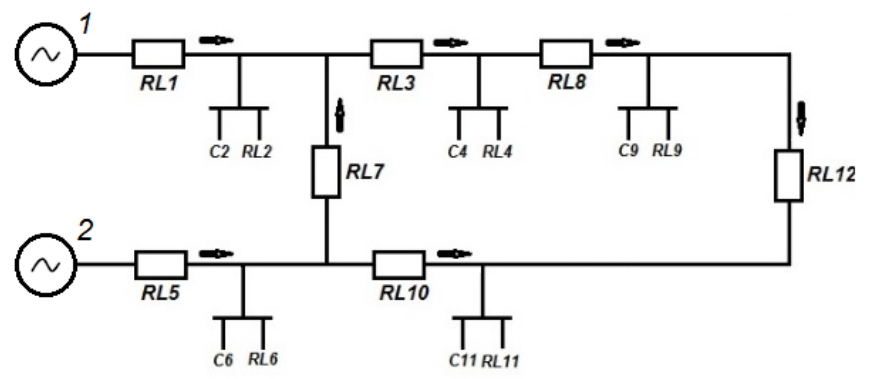

Fig. 2. Electric circuit for calculation.

The following rules were adopted when drawing up the circuit:

- the conditional direction of the current flow in the chains, which is necessary for composing the calculation equations, is shown by arrows;

- the active-inductive load is indicated by the symbols "RL" with the corresponding number;

- the capacitive load is indicated by the symbol "C" with the corresponding number.

The model of the power supply circuit was considered in a linear setting.

The circuit is described by a system of 17 differential equations that include the balance of currents in the circuit junctions and the balance of voltage drops in the circuits.

The simulation was performed using numerical methods. The calculation system of equations was reduced to the Cauchy form with the selection of the desired unknowns in the left part of the equations, namely, currents in chains with active-inductive load and voltages in chains with capacitive load. The standard Runge-Kutt 4-order calculation algorithm with automatic step selection was used.

The transition process was calculated based on zero initial conditions: at the time of synchronous connection of AC generators to the power grid, the initial currents in the chains are absent.

The load value of one of the consumers had stepwise changes after connecting the generators to the network after a certain period of time required to obtain a steady state.

\section{Results and discussion}

The cost of electricity produced by each producer is individual. Accurate economic calculations require knowledge of the true picture of the flows of transmitted capacity across all elements of the power system, taking into account the loads and parameters of the circuit as the source information. 

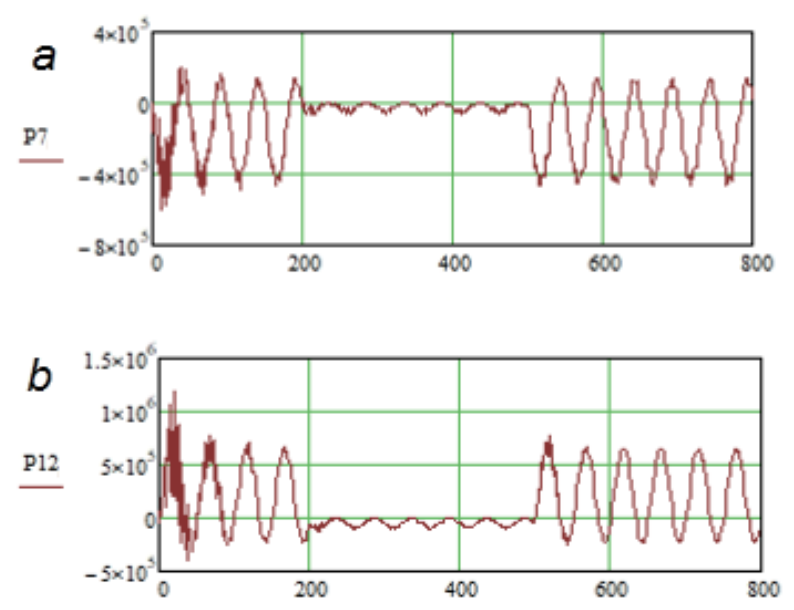

Fig. 3. Waveforms of power changes in chains of the power supply circuit.

The state of the multi-junction circuit was modeled as a numerical experiment (Fig. 2) when the load of consumers changes. The value of transmitted power as the result of instantaneous values of current and voltage was the evaluation criterion. The disturbing influence on the established mode of operation of the power system was provided by a stepwise change in the load of the consumer in the RL11 chain.

Taking into account the variable nature of the current, the direction of power transmission is determined by the sign of the average value of sinusoidal power oscillations. The load change in the RL11 chain resulted in a change in the power transfer direction in the RL12 chain (Figure 3b), while the power transfer direction remained the same in the RL7 chain (Figure 3 a)

The process of transferring power over time through the chain RL12 occurred according to the following algorithm: until with a mark "200" power is transmitted from the 2nd source; between the marks "200" and "500" power is transmitted from the 1st source, and from the time marked "500" power came from the 2nd source too.

Changes in the power flow pattern are caused by changes in the load of consumers. In this case, the parameters of power supply circuits begin to play a significant role. These parameters also appear in economic calculations if we take into account possible differences in the cost of electricity from producers.

\section{Conclusion}

Modeling of transients in power supply systems makes it possible to get an objective picture of events. As a result of changes in the load, there may be a balance of flows of transmitted electrical power, in which electric power from different sources will flow through a particular element of the ring. This information may be required for economic justification of the operation of power supply systems and for specific calculations with consumers of electric energy.

\section{References}

1. IRENA, Electricity Storage and Renewables: Costs and Markets to 2030, International Renewable Energy Agency, Abu Dhabi (2017) 
2. Electric Energy Storage Technology Options: A White Paper Primer on Applications, Costs, and Benefits. EPRI, Palo Alto, CA: 1020676 (2010)

3. Benefit Analysis of Energy Storage: Case Study with the Sacramento Utility Management District.EPRI, Palo Alto, CA:1023591 (2011)

4. S. Kumar, S.K. Goyal, A Particle Swarm Optimization for Reactive Power Optimization International Journal of Computational Engineering Research (IJCER), 440-46 (2014)

5. G. Sahu, K. Swarnkar, Review of Reactive Power Optimization Using Evolutionary Computation Techniques Advance in Electronic and Electric Engineering 4, 73-82 (2014)

6. B. Mandal, Optimal reactive power dispatch using quasi-oppositional teaching learning based optimization Electrical Power and Energy Systems 53 123-34 (2013)

7. V. Patel, R. Patel, Reactive Power Management through Dispatch Analysis using Evolutionary Algorithms International Journal of Innovative Technology and Exploring Engineering (IJITEE) 8 759-65 (2019)

8. S. Mishra, D. Das, Distribution System Load Flow Methods: A Review. IEEE Vol.04 № 07 7-25 (2008)

9. V. Parmar, M. Shah, Load Flow and Voltage Instability Analysis Using MI Power Software. IJESI.Vol. 7, №6, 49-55 (2018)

10. I. Shulgin, A. Gerasimenko, Q.Z. Su, Stochastic Simulation of Covariance Matrix and Power Load Curves in Electric Distribution Networks. JSFUET Vol 5 № 1 39-56 (2012) 